\title{
Albertine, en cinq temps masculinisée? Regard féminin/féministe sur la traduction polonaise
}

\author{
Aleksandra Chrupała \\ Joanna Warmuzińska-Rogóż \\ Université de Silésie \\ «Il faut que la femme se mette au texte \\ - comme au monde, \\ et à l'histoire - de son propre mouvement. »
}

(Cixous 39)

En 2010, les amateurs de théâtre québécois en Pologne, qui jusqu'à présent n'ont pu connaitre que la traduction de Bousille et les justes de Gratien Gélinas et de deux pièces de théâtre de Michel Tremblay ${ }^{1}$, ont reçu la version polonaise d'Albertine, en cinq temps. La représentation scénique de Piéć rasy Albertyna a vu le jour au Theâtre de Silésie à Katowice, où le metteur en scène, Gabriel Gietzky, a monté la pièce traduite vers le polonais par Jacek Mulczyk-Skarżyński. La représentation scénique à laquelle nous avions assisté nous a poussé à réfléchir sur des enjeux intéressants entre les genres, aussi bien au niveau de l'auteur et de son personnage, de l'auteur et du traducteur que de ce dernier et de sa langue d'arrivée ou bien de sa langue maternelle. Plus que toute autre, la pièce tremblayenne nous fait penser à la perspective de Jacques Derrida selon qui on ne peut pas se situer en dehors du discours phallogocentrique, c'est-à-dire celui qui impose la perception des notions-clés comme le «sexe » et le « féminin/ masculin » par le biais de la domination masculine ${ }^{2}$.

Il va sans dire que notre regard, quoique critique, n'a pas pour but de pointer du doigt les imperfections de la traduction polonaise d'Albertine, en cinq temps. Nous recourons à un regard double : à la fois féminin et subjectif, sur le texte choisi, tout en ayant à l'esprit l'opinion de Sherry Simon selon qui « language intervenes actively in the creation of meaning. Like other forms of representation, language does not simply "mirror" reality; it contributes to it» (8). Notre analyse se présente donc comme une réflexion socio-culturelle découlant du sexe de l'auteur et du traducteur (Maliszewski 152), qui s'impose incontestablement dans le contexte des enjeux liés à la question du féminin et du masculin. Nous suivons par là la conception de la traduction féministe empruntée notamment par Luise von Flotow, qui la définit comme : «(..) an approach to translation that has appropriated and adapted many of the techniques and theories that underlie the writing it translates. » (von Flotow 74, c'est nous qui soulignons). Autrement dit, notre réflexion recourt à la pensée féministe dans la mesure où elle

\footnotetext{
${ }^{1}$ La traduction polonaise du drame Bousille et les justes de Gratien Gélinas (dont le titre polonais est Sami porzadni ludzie, trad. Jerzy Lisowski, Marta Skibniewska) a été publiée dans la revue littéraire «Dialog» en 1979 (numéro 2). Les Belles-søurs, dont le titre polonais est Siostrayczki, est parue dans le numéro 8 de la même revue en août 1990. Quant à la traduction du V rai monde dont le titre polonais est $W$ najlepszej wierze, elle a été publiée dans le numéro 11 en novembre 1994. Les deux pièces ont été traduites par Józef Kwaterko. À notre connaissance, contrairement aux autres traductions, Les Belles-søurs a déjà été amplement analysée, notamment par Janusz Przychodzeń (2000).

2 Pour les enjeux entre le logocentrisme et le phallogocentrisme, voir: Derrida 1967, 1978
} 
s'efforce de démontrer comment la langue peut être envahie par un locuteur masculin, nous remontons donc à l'origine de la pensée féministe, avec cette différence que nous changeons un peu le schéma décrit amplement par les féministes; schéma selon lequel il faut s'opposer au «langage patriarchal $»^{3}$, notamment grâce à la traduction. Nous proposons d'analyser le phénomène de la «traduction masculinisante » qui perturbe le langage féminin tout en soulignant que ce n'est qu'une lecture possible parmi beaucoup d'autres. Si l'on revient à la pensée derridienne, nous avons l'intention de vérifier comment, dans la traduction masculine du langage féminin, se reflète le langage patriarchal incarnant la force phallique.

\section{Albertine - femme québécoise créée par un homme}

La pièce tremblayenne met au centre Albertine, une femme que le lecteur/spectateur observe pendant les cinq moments cruciaux de sa vie, les cinq «âges » d'Albertine (Camerlain 125), incarnés dans le texte par les cinq personnages. Comme le fait remarquer Eza Paventi, «Michel Tremblay a donné à son personnage cinq incarnations, cinq voix qui ignorent la linéarité du temps pour vivre en cœur une vie »(70). Or, le personnage féminin, multiplié par cinq et confronté à une autre femme, Madeleine, sa sœur, prend naissance grâce à l'auteur-homme. Cependant l'auteur souligne lui-même dans différents entretiens avoir vécu une enfance heureuse, entouré de nombreuses femmes (David, Lavoie 11). De toute évidence, élevé dans un univers presqu'exclusivement féminin, M. Tremblay possède une grande familiarité avec celui-ci. L'un des outils dont il se sert à cette fin est certainement la langue. Lorsqu'on l'interroge sur le langage spécifique que parlent ses personnages, l'auteur a toujours expliqué que le joual était pour lui la langue des femmes québécoises. Nous considérons donc que M. Tremblay dépeint parfaitement « l'inconcient collectif féminin universel» (Ruprecht 6), même si dans Albertine, en cinq temps qui se démarque par «la polyphonie de monologues» (Godin 181), il transmet et transfigure le langage de l'héroïne à travers le filtre de la conscience masculine. On ne peut pas non plus passer sous silence le fait que le monde romanesque et théâtral de M. Tremblay est un monde féminin en ce sens qu'il n'est habité que par des femmes et des homosexuels parlant d'eux-mêmes au féminin. Paulette Collet souligne que l'absence des personnages masculins est frappante et significative car selon les dires mêmes de l'écrivain « [i]l n'y a pas d'hommes au Québec » (cité par Collet 600). Albertine est donc une femme qui incarne l'histoire des femmes et l'histoire de l'humanité et dans laquelle certains critiques voient "l'image du Christ devenu la femme dans sa globalité, la femme mythique» (Ruprecht 6). Bien que, de premier abord, nous ayons affaire à un renversement de modèle caracterisé déjà par Marguerite Duras. Selon l'auteure, l'homme, contrairement à la femme, n'est pas obligé d'effectuer « une traduction » car il travaille avec le langage qui lui est propre (cité par Osadnik 92) en ce sens que c'est l'homme qui se donne pour tâche de transmettre le langage des femmes. Pour notre part, il nous semble que le langage féminin chez M. Tremblay n'est pas perturbé, biaisé, voire même masculinisé. Or, le texte tremblayen imprégné du caractère féminin des personnages, notamment au niveau de leur manière de parler, ce que nous verrons plus loin, est soumis à la traduction du traducteur-homme qui fait paraittre la version polonaise d'Albertine, en cinq temps. Quelles sont les conséquences de cet itinéraire de création-traduction? Autrement dit, peut-on parler dans le cas de la traduction

\footnotetext{
${ }^{3}$ Pour la description du contexte de l'apparition de la traduction féministe voir: von Flotow 1991.
} 
polonaise d'un langage masculin dévoilé dans un costume féminin ? Pour étudier les relations et interdépendances dans le schéma ci-dessus et vérifier leur pertinence dans le cas de Pię́ rasy Albertyna, nous proposons de centrer notre réflexion sur la notion de fidélité qui, confrontée à la réflexion sur l'influence du sexe de traducteur sur son activité, met en jeu des relations fort complexes entre l'original et la traduction ainsi que parmi les acteurs qui participent à l'opération traduisante, et ceci dans le cadre de la métaphore renvoyant à des enjeux entre féminin et masculin.

\section{Les enjeux : traducteur - langue maternelle}

Suivant la conception décrite notamment par Lori Chamberlain dans son essai "Gender and the Metaphorics of Translation », nous tenterons de vérifier la relation entre l'auteur de la traduction (dans notre cas : un homme) et la langue vers laquelle il traduit (langue maternelle) (Chamberlain 392) ${ }^{4}$. Cette perspective fait incontestablement penser à un aspect typiquement langagier.

\section{Un SYSTÈME, deux PARLERS ?}

On peut se demander dans quelles mesures les hommes et les femmes parlent ou non la même langue. Les recherches portant sur les différences entre les parlers des hommes et des femmes ont commencé dans les années 1970, suscitées par l'intérêt concernant le problème de la discrimination des femmes qu'on observait à l'époque. La publication, en 1975, de l'ouvrage de Robin Lakoff Language and Woman's Place a déclenché une véritable avalanche de titres sur la question ${ }^{5}$. Il est intéressant de souligner que ces études concernaient en majorité le parler des femmes; les analyses du parler des hommes étaient (et le sont toujours) beaucoup plus rares. Ce déséquilibre peut s'expliquer par deux faits :

- premièrement, ce type de recherches exige qu'on se concentre sur les traits constants et communs, et qu'on néglige tout ce qui a un caractère individuel, momentané et passager. Or, il s'avère que les hommes privilégient la fonction représentative de la langue et se servent de préférence d'éléments linguistiques neutres. En tant que groupe, ils ne possèdent pas beaucoup de traits particuliers qui soient stables, généralisés et propres à eux. Parmi les caractéristiques attribuées généralement aux hommes on distingue les vulgarismes, les jurons et l'obscénité, mais ce n'est qu'une image simplifiée et stéréotypée. Ces comportements langagiers ne sont pas exclusivement masculins. ${ }^{6}$ La parole des femmes, qui préfèrent la fonction expressive de la langue est au contraire caractérisée par tout un éventail de trais expressifs typiquement féminins (Handke 19);

\footnotetext{
${ }^{4}$ Par contre, compte tenu de l'étendue restreinte de la présente analyse, nous ne nous pencherons pas sur une relation entre la traduction (femme) et l'original, et surtout son auteur (homme), détronisé et remplacé par le traducteur - autrement dit l'auteur de la traduction, en l'occurence l'homme (Cf. Chamberlain).

5 Voir par exemple Labov 1976, Yaguello 1978, Trudgill 1983, Cameron 1985, Chambers 1995.

${ }^{6}$ Quant à la réalité polonaise, les résultats de différentes enquêtes témoignent du fait que dans ces derniers temps la manière d'être des femmes a beaucoup changé. Les interrogés soulignent surtout une augmentation considérable de comportements agressifs, qui s'expriment principalement dans la langue, en particulier dans l'emploi de vulgarismes (cf. p.ex. IMAS - Report 2006).
} 
- deuxièmement, dans ce type de recherches c'est toujours le langage des hommes qui est pris pour norme, tandis que celui des femmes est considéré comme défiance ou comme défaut. Et, comme le constate C. KerbratOrecchioni, la curiosité des linguistes se porte plus sur les pratiques discursives considérées comme "marquées» que sur les discours admis comme «non marqués » (Aebischer et Forel 12, cité par Kerbrat-Orecchioni 37-38).

Les différences en question sont-elles tellement importantes pour que l'on puisse parler de deux langues différentes? Les résultats des recherches démontrent qu’il est difficile d'y voir deux systèmes distincts; la LANGUE saussurienne est en effet stable et commune à tous les humains. Les divergences éventuelles ne se manifestent que dans la PAROLE, c'est pour cela qu'on préfère souvent le terme «style propre aux femmes » à celui de la «langue des femmes».

Qu'est-ce qui est à l'origine de ces variations? Les chercheurs proposent des réponses différentes. Sans entrer dans les détails, nous nous contenterons de reprendre leur typologie (cf. Kerbrat-Orecchioni 64-70), tout en restant fidèles au terme STYLE /PARLER féminin.

- LE BIOLECTE - explication bio-psychologique souligne l'influence du sexe et de l'âge de la personne qui parle ainsi que le rôle d'autres facteurs naturels (système hormonal, différence dans l'appreil phonatoire...)

- LE PSYCHOLECTE - explication psychologique ou psychanalytique précise que les styles masculin et féminin appartiennent à deux psychès différentes

- LE SOCIOLECTE - explication en termes de status représente une vision plutôt négative selon laquelle le style féminin reflète l'infériorité de la position sociale réservée à la femme

- LE GENDERLECT ${ }^{7}$ - explication en termes de gender attribue les caractéristiques de style féminin aux rôles réservées à la femme dans la société; dans cette conception ce n'est pas le sexe en tant que tel qui est déterminatif, mais l'élaboration sociale du sexe qu'on appelle souvent « gender».

\section{Les hommes disent, les femmes parlent}

Parmi les caractéristiques du parler des femmes, les linguistes citent le plus souvent (Cf., Kerbrat-Orecchioni 44-60 et Handke 19-20) :

- un débit plus rapide (les femmes parlent en moyenne plus vite que les hommes; elles contruisent généralement des phrases plus longues que les hommes)

- un caractère émotionnel (les femmes sont supposées être plus portées à la tristesse que les hommes)

- une plus grande expressivité (on appelle cette langue « orientée sur la personne », tandis que la langue des hommes est plutôt « orientée

\footnotetext{
7 Nous gardons le terme anglais, car la forme adaptée au français (genderlecte) est beaucoup plus rare. D'ailleurs, le mot gender lui-même pose des problèmes aux terminologues français (cf. p.ex. Hay 2002 ou Journal officiel du 22 juillet 2005 de la Commission générale de terminologie et de néologie).
} 
sur les objets »); ce style est plus riche en moyens d'expression positifs et négatifs, quoique ceux-ci soient moins fréquents. En polonais, il s'agit de :

- diminutifs, augmentatifs en fonction d'hypocristiques,

- exclamations, interjections,

- formes d'adresse variées,

- $\quad$ synonymes, néologismes

- euphémismes

- augmentatifs, diminutifs marqués,

- exclamations, interjections,

- vulgarismes et jurons (surtout dans les apostrophes)

- un caractère évaluatif positif ou négatif qui s'accompagne souvent d'une sorte de redondance textuelle (surabondance de possessifs et de démonstratifs, d'adjectifs et d'adverbes, emploi fréquent d'expressions phraséologiques)

- la facilité d'employer la narration à la première personne; les hommes ne sont pas prêts à se "confier», d'où peut-être leur propension à éviter le « je » dans les conversations

Après avoir présenté brièvement les différences entre les parlers masculin et féminin, nous nous proposons d'identifier le style adopté par le traducteur. Nous éloignant un peu de l'axe de recherches suivi notamment par Janusz Przychodzen (2000) qui analysait les différentes stratégies adoptées par le traducteur afin de rendre compte du joual en polonais ${ }^{8}$, nous choisirons d'examiner si celui-ci tente de reproduire la spécificité du parler des personnages féminins mis sur la scène par M. Tremblay. C'est à cet effet, que nous avons préparé une juxtaposition de quelques fragments de l'original et de la version polonaise afin d'illustrer l'analyse du texte en fonction de la grille de lecture présentée ci-dessus.

\section{Les phrases construites par les femmes sont plus longues que celles des hommes}

Le texte de la traduction polonaise est plus «haché » que celui de la version originale. Néanmoins il faut avoir à l'esprit que la pièce est destinée à être interprétée par des actrices, qui peuvent toujours modifier et adapter si nécessaire l'intonation".

\begin{tabular}{|l|l|l|l|}
\hline $\begin{array}{c}\mathbf{N}^{\mathbf{0}} \\
\text { de } \\
\text { page }\end{array}$ & \multicolumn{1}{|c|}{ Texte original } & $\begin{array}{c}\mathbf{N}^{\mathbf{o}} \\
\text { de } \\
\text { page }\end{array}$ & \multicolumn{1}{|c|}{ Texte de la traduction } \\
\hline 77 & $\begin{array}{l}\text { Je me suis habillée en neuf, du pas cher } \\
\text { mais du beau, pis chus partie me } \\
\text { chercher une job! }\end{array}$ & 34 & $\begin{array}{l}\text { Kupiłam sobie nowe ubrania. } \\
\text { Tanie, ale ładne. I poszłam } \\
\text { szukać pracy! }\end{array}$ \\
\hline
\end{tabular}

${ }^{8}$ C'est la question que nous développons dans un autre texte : Femmes, joual et figure $d u$ traducteur - version polonaise d'Albertine, en cinq temps de Michel Tremblay (à paraître).

${ }^{9}$ Nous tenons à remarquer aussi que les traducteurs de pièces de théâtre sont parfois obligés de suivre les recommandations des metteurs en scène qui leur demandent de raccourcir les phrases ou de les ponctuer, afin d'adapter le texte aux besoins de la représentation scénique. 


\begin{tabular}{|l|l|l|l|}
\hline 83 & $\begin{array}{l}\text { Ben voyons donc, c'est toi qui as } \\
\text { raison, sont toutes pareils, les hommes, } \\
\text { y finissent toujours par nous avoir! }\end{array}$ & $\begin{array}{l}\text { No proszę. To właśnie ty masz } \\
\text { rację. Oni wszyscy sa tacy sami. } \\
\text { Faceci. Zawsze nas } \\
\text { wykorzystaja! }\end{array}$ \\
\hline 86 & $\begin{array}{l}\text { Quand les deux p'tits pinceaux de } \\
\text { lumières vont tourner le coin de la } \\
\text { route, tout à l'heure, dites-vous bien } \\
\text { que j’vas être parfaitement heureuse... } \\
\text { pis que je veux pas en discuter. }\end{array}$ & $\begin{array}{l}\text { Kiedy dwa snopki światel } \\
\text { skręca za chwile na rogu drogi. } \\
\text { Wierzcie, ze będę } \\
\text { najszczésliwszą kobietą na } \\
\text { świecie... I ze nie chcę o tym } \\
\text { dyskutować. }\end{array}$ \\
\hline 91 & $\begin{array}{l}\text { Quand je vois Thérèse arriver, les } \\
\text { lendemains de la veille, poquée pis } \\
\text { encore éméchée, trop fine avec moi } \\
\text { parce qu'a' se sent coupable d'avoir bu } \\
\text { mais quand même baveuse parce que } \\
\text { c'est sa seule façon de me monter son } \\
\text { indépendance, ... }\end{array}$ & $\begin{array}{l}\text { Jak wraca rano, na kacu... } \\
\text { Milutka, bo ma poczucie winy, } \\
\text { że piła. Ale przy tym bezczelna, } \\
\text { bo wie, że wódka to jedyny } \\
\text { sposób by pokazać mi swoja } \\
\text { niezależność... }\end{array}$ \\
\hline
\end{tabular}

\section{Le style des femmes est riche en moyens d'expression positifs et négatifs}

Comme on pouvait s'y attendre, le nombre des diminutifs et augmentatifs est plus important dans le texte de la traduction que dans l'original. En effet le choix des formes diminutives et augmentatives ainsi que les possibilités de les créer sont infiniment plus grandes en polonais qu'en français : le polonais permet de former des diminutifs de cinq catégories morphologiques, y compris les pronoms (démonstratifs - «samiutki», indéfinis - "kaq̇driuteńki», "wsyyściutki») et, dans une moindre mesure, les verbes ("plakusiać»), les adverbes ("bielusieńko»). Ils peuvent également assumer des fonctions différentes: indiquer les petites dimensions ou le manque d'importance de l'objet donné, exprimer un sentiment de tendresse et d'affection envers l'objet donné ou bien envers la personne à laquelle on s'adresse et finalement exprimer une attitude méprisante ou ironique envers l'objet en question. La plupart des formes diminutives qui apparaissent dans la traduction assument justement cette dernière fonction (par exemple: "męzulek», "charakterek»). C'est uniquement l'hypocoristique du prénom «Albertine» («Bercia») qui joue effectivement son rôle. En plus, certains emplois de formes diminutives nous semblent discutables, comme le mot kobitki, qui peut être pourvu d'une connotation péjorative ou condescendante, sans que le texte de départ ne le suggère.

\begin{tabular}{|c|c|c|c|}
\hline $\begin{array}{l}\mathrm{N}^{\mathrm{o}} \mathrm{de} \\
\text { page }\end{array}$ & Texte original & $\begin{array}{l}N^{o} \text { de } \\
\text { page }\end{array}$ & Texte de la traduction \\
\hline 89 & $\begin{array}{l}\text { Ferme les yeux, on va essayer de } \\
\text { s'imaginer qu'on est là... La vois-tu, } \\
\text { au fond, là-bas? Une p'tite lumière } \\
\text { qui brille au bord de la nuit? Ç'a } \\
\text { l'air tellement paisible, de loin... pis }\end{array}$ & 41 & $\begin{array}{l}\text { Zamknij oczy. Spróbujemy sobie } \\
\text { wyobrazić, że tam jesteśmy... } \\
\text { Widzisz, w dali, tam? Światełko, } \\
\text { które pali się na skraju nocy? } \\
\text { Wygląda tak miło, z daleka... I jest }\end{array}$ \\
\hline
\end{tabular}




\begin{tabular}{|l|l|l|}
\hline $\begin{array}{l}\text { plaisant. Y'a deux femmes, } \\
\text { su'a'galerie. J'me demande de quoi } \\
\text { y peuvent ben parler. }\end{array}$ & $\begin{array}{l}\text { miłe. Dwie kobitki. Na ganku. } \\
\text { Zastanawiam się o czym to one } \\
\text { moga gadać. }\end{array}$ \\
\hline
\end{tabular}

Le fonctionnement des augmentatifs est identique en ce sens qu'ils peuvent exprimer soit du dédain (surtout avec un changement de genre grammatical, par exemple: "dziewcryna», "dziewnsyysko»), soit une attitude affectueuse (par exemple "pies», «psisko»). Il s'avère que ce dernier emploi n'est pas exemplifié dans le texte examiné; nous n'avons trouvé que des formes à valeur plutôt négative, telles que : "fryty», «ciuchy», «buta». Quant aux formes d'adresse, elles ne sont nombreuses ni dans l'original ni dans la traduction, nous en avons répéré quelques-unes comme : "Wybacz, złotko »,-ale.... », "Daj spokój, stara..”. Il en est de même avec les néologismes : une seule forme retrouvée «kosmokombinator» (scaphandrier).

Parmi les propriétés du parler féminin, on mentionne souvent une certaine prédilection pour les euphémismes. Cela concerne les deux langues traitées, mais à des degrés différents. Comparativement au français le polonais recourt moins souvent à des formes atténuées. Au lieu de périphrases élégantes, il se sert souvent de mots forts, truculents, même s'ils possèdent des connotations péjoratives, par exemple : "nieślubny syn» vs «fils naturel»; «samochód uínwany» vs «voiture d'occasion». Cela peut probablement justifier l'emploi de formes polonaises plus fortes au lieu des expressions atténuées qu'on trouve dans l'original. Il est à remarquer que la traduction fidèle à l'original aurait même pu rendre le texte artificiel.

\begin{tabular}{|l|l|l|l|l|}
\hline $\begin{array}{c}\mathbf{N}^{\mathbf{0}} \text { de } \\
\text { page }\end{array}$ & \multicolumn{1}{|c|}{ Texte original } & $\begin{array}{l}\boldsymbol{N}^{\mathbf{v}} \\
\text { de } \\
\text { page }\end{array}$ & Texte de la traduction & $\begin{array}{l}\text { Traduction } \\
\text { littérale du } \\
\text { polonais } \\
\text { en français }\end{array}$ \\
\hline 18 & $\begin{array}{l}\text { Parce que j'vas être bien, ici. } \\
\text { (Court silence. }) \text { Même si ça } \\
\text { sent pas bon. }\end{array}$ & 3 & $\begin{array}{l}\text { Bo będzie mi tu dobrze. } \\
\text { (cisza) Nawet jeśli tu } \\
\text { śmierdzi. }\end{array}$ & $\begin{array}{l}\text { Parce que } \\
\text { j’vais être } \\
\text { bien, ici. } \\
\text { silence. }) \\
\text { Même si ça } \\
\text { pue ici. }\end{array}$ \\
\hline 70 & $\begin{array}{l}\text { Non, ce n'est pas de la folie. } \\
\text { C'est de l'ignorance. }\end{array}$ & 31 & $\begin{array}{l}\text { Nie, to nie szaleństwo. To } \\
\text { głupota. }\end{array}$ & $\begin{array}{l}\text { Non, ce } \\
\text { n'est pas de } \\
\text { la folie. } \\
\text { C'est de la } \\
\text { stupidité. }\end{array}$ \\
\hline
\end{tabular}

Ce qui nous parait plus intéressant, c'est l'emploi d'expressions assez fortes, témoignant en quelque sorte de la rudesse du langage adopté par le traducteur. Certaines recherches démontrent que la tendance à employer des formes explicites, directes et plus marquées au lieu d'expressions euphémiques ou périphrases élégantes est plus caractéristique pour 
les traducteurs-hommes ${ }^{10}$. C'est peut-être pour cette raison qu'on trouve dans le texte de traduction des séquences telles que : « być spasionym » ou «choćby skały sraty ».

\begin{tabular}{|c|c|c|c|c|}
\hline $\begin{array}{l}\mathrm{N}^{\mathrm{o}} \mathrm{de} \\
\text { page }\end{array}$ & Texte original & $\begin{array}{l}N^{o} \\
\text { de } \\
\text { page }\end{array}$ & Texte de la traduction & $\begin{array}{l}\text { Traduction } \\
\text { littérale du } \\
\text { polonais } \\
\text { en français }\end{array}$ \\
\hline 85 & $\begin{array}{l}\text { On te l'enlèvera pas, ta } \\
\text { merveille... } \\
\text { Ah! Seigneur, non! }\end{array}$ & 39 & $\begin{array}{l}\text { Nie martw się. Nie } \\
\text { odbijemy ci twojego } \\
\text { supermena... } \\
\text { O nie! Choćby skały srały! }\end{array}$ & $\begin{array}{l}\text { On te } \\
\text { l'enlèvera } \\
\text { pas, ton } \\
\text { superman } \\
\text { Oh non! } \\
\text { Même si les } \\
\underline{\text { rocs }} \\
\underline{\text { chiaient! }}\end{array}$ \\
\hline 87 & $\begin{array}{l}\text { Quand t'es partie, j'ai perdu } \\
\text { ma seule confidente... Le } \\
\text { téléphone a pus sonné... [...] } \\
\text { Pis quand tu m'as emmené } \\
\text { ta petite-fille pour la } \\
\text { première fois pis que j'ai vu } \\
\text { à qul point t'avais engrassé... } \\
\text { j'ai tellement ri! C'est peut- } \\
\text { être pour ça, tant qu'à ça, } \\
\text { que j’ai de la misère à me } \\
\text { rappeler de toi... Les } \\
\text { dernières fois que je t'ai vue } \\
\text { t'étais tellement grosse... }\end{array}$ & 40 & $\begin{array}{l}\text { Kiedy odeszłaś, straciłam } \\
\text { jedyna przyjaciółkę... } \\
\text { Telefon nie dzwonił... [...] I } \\
\text { kiedy za pierwszym razem } \\
\text { przyszłaś do mnie ze swoją } \\
\text { wnuczka, i gdy zobaczyłam } \\
\text { jak bardzo przytyłaś... } \\
\text { Tak się uśmiałam! To może } \\
\text { też dlatego nie mogę sobie } \\
\text { ciebie przypomnieć... } \\
\text { Ostatnimi razy kiedy cię } \\
\text { widziałam. Byłaś taka } \\
\text { spasiona... }\end{array}$ & $\begin{array}{l}\text { Les } \\
\text { dernières } \\
\text { fois que je } \\
\text { t'ai } \\
\text { vue, } \underline{\text { t'étais }} \\
\underline{\text { tellement }} \\
\underline{\text { bouffie... }}\end{array}$ \\
\hline
\end{tabular}

Ce qui peut être considéré comme un autre indice de la brutalisation du texte traduit, c'est la forte présence de vulgarismes et de jurons, plus nombreux et plus variés dans la version polonaise que dans l'original. Cependant, il nous faut avouer que les avis sur la question des vulgarismes dans le parler féminin sont partagés : certains spécialistes (cf., W. Labov, N. Huston) considèrent que les femmes adoptent un niveau de langue correct (parfois même hypercorrect), surveillent davantage leur langage et préfèrent éviter les jurons ou les mots grossiers; d'autres chercheurs estiment au contraire que les femmes s'en servent fréquemment, pour mieux manifester l'expressivité qui leur est

${ }^{10}$ Voir en particulier Bednarczyk 40, qui parle à ce titre de la brutalisation du texte traduit. 
propre, surtout dans les apostrophes. Les uns et les autres constatent cependant une évolution récente, à savoir le fait que les jeunes filles et les jeunes femmes font actuellement usage des formes de plus en plus vulgaires non seulement pour accentuer de vives émotions, mais apparemment sans raison, juste pour affirmer leur façon d'être. C'est peut-être cette observation qui a poussé le traducteur à augmenter considérablement le nombre et l'éventail des vulgarismes et jurons dans la traduction.

\begin{tabular}{|c|c|c|c|}
\hline $\begin{array}{c}\mathbf{N}^{\circ} \\
\text { de } \\
\text { page }\end{array}$ & Texte original & $\begin{array}{l}\mathbf{N}^{\mathrm{o}} \\
\text { de } \\
\text { page }\end{array}$ & Texte de la traduction \\
\hline 15 & C'est ben pour dire, hein... & 2 & $\underline{\text { Kurde, ciężko w to uwierzyć }}$ \\
\hline 25 & $\begin{array}{l}\text {..., parce que celui-là est pas } \\
\text { vergeux! }\end{array}$ & 7 & ..., bo ten jest do dupy! \\
\hline 28 & $\begin{array}{l}\text { Arrête donc de dire des } \\
\text { niaiseries... }\end{array}$ & 9 & Nie pierdziel głupot... \\
\hline 65 & $\begin{array}{l}\text { Un homme, Medeleine, pas } \\
\text { un p'tit morveux de son âge } \\
\text { qui se serait contenté de } \\
\text { l'embrasser avec la bouche } \\
\text { fermée, là, un homme faite! }\end{array}$ & 28 & $\begin{array}{l}\text { Faceta, Madeleine! Nie jakiegoś gówniarza } \\
\text { w jej wieku, któremu wystarczyłoby ja } \\
\text { pocałować z zamkniętymi ustami! Nie, } \\
\text { dorosłego faceta! }\end{array}$ \\
\hline 80 & $\begin{array}{l}\text { Essaye de parler avec } \\
\text { Thérèse, tu vas voir } \\
\text { comment c'est agréable! }\end{array}$ & 36 & $\begin{array}{l}\text { Spróbuj porozmawiać z z Thérèse, } \\
\text { zobaczysz jakie to } \underline{k \ldots} \text {... przyjemne! }\end{array}$ \\
\hline 81 & Arrête donc de t'en faire! & 37 & Przestań już się obwiniać, do cholery! \\
\hline 92 & $\begin{array}{l}\text { Y m'écoutent pas, pourquoi } \\
\text { j’les écouterais? }\end{array}$ & 43 & $\begin{array}{l}\text { One mnie maja } w \text { dupie, więc } z \text { jakiej racji } \\
\text { ja miałabym je wysłuchiwać? }\end{array}$ \\
\hline 98 & $\begin{array}{l}\text { C'est toujours comme ça } \\
\text { qu'on s'est fait avoir, pis on } \\
\text { n'a pas encore appris! }\end{array}$ & 46 & $\begin{array}{l}\text { Właśnie tak dajemy sie zawsze wydymać } \mathrm{i} \\
\text { nigdy niczego się nie uczymy! }\end{array}$ \\
\hline
\end{tabular}

Une lecture attentive du texte traduit permet de constater une différence non seulement quantitative mais aussi qualitative dans l'emploi des vulgarismes et jurons. Dans la version originale, il y a au total huit emplois de sacres (« maudit» 4 fois et «sacré » 4 fois) auxquels s'ajoutent trois emplois du nom de Dieu («Dieu » 2 fois et «Seigneur» 1 fois). Dans la version traduite, nous avons répéré dix-huit emplois de mots plus ou moins vulgaires ("kurcze», "kurde», «kurwa», "cholera», "dupa», "dupowaty», "piepryony», "pierdoly», "pierdzieli», "gówniarz», "szllag», "wydymać»), accompagnés de quatre emplois du nom «Bóg» et «diabet» (ou bien des variantes «boski» et «diabelski»). Un nombre élevé de jurons polonais témoigne peut-être de la volonté du traducteur de bien refléter l'une des particularités du joual, c'est-à-dire une forte présence de sacres, néanmoins le choix des formes peut prêter à discussion. En effet, le polonais dispose de toute une gamme de jurons liés à la religion catholique, qui sont utilisés d'une manière plus ou moins blasphématoire et, qui plus est, sont considérés 
comme caractéristiques pour le parler féminin, par exemple: "Matko kochana», "O Jezu», « Jezusie Nazarejski », "Jezus Maria », « na litość boskq», etc.

\section{Le style des femmes se caractérise par une surabondance de possessifs, démonstratifs, adjectifs, adverbes, expressions phraséologiques}

On considère que le caractère évaluatif, positif ou négatif, propre au style féminin s'accompagne souvent d'un emploi massif de certains pronoms, adjectifs, adverbes et expressions phraséologiques. Tous ces marqueurs se trouvent dans la traduction mais à des degrés différents. Nous avons repéré plusieurs exemples où la forme du pronom possessif est redoublée en quelque sorte par le pronom possessif et démonstratif. Ce type de constructions est caractéristique du langage parlé, il sert à souligner une attitude émotionnelle (très souvent négative) du locuteur envers l'interlocuteur ou bien envers l'objet dont il parle.

\begin{tabular}{|c|c|c|c|c|}
\hline $\begin{array}{l}\mathrm{N}^{\circ} \mathrm{de} \\
\text { page }\end{array}$ & Texte original & $\begin{array}{l}N^{o} \text { de } \\
\text { page }\end{array}$ & Texte de la traduction & $\begin{array}{l}\text { Traduction } \\
\text { littérale du } \\
\text { polonais en } \\
\text { français }\end{array}$ \\
\hline 46 & $\begin{array}{l}\text { Pis quand j'te vois } \\
\text { venir te pavanner ici } \\
\text { avec ton mari qui a } \\
\text { réussi pis tes enfants } \\
\text { super-bright! }\end{array}$ & 18 & $\begin{array}{l}\text { I jak cię widzę, kiedy się tutaj } \\
\text { puszysz z tym twoim } \\
\text { mężulkiem, któremu się } \\
\text { udało i z tymi twoimi } \\
\text { dziećmi z czerwonym } \\
\text { paskiem! }\end{array}$ & $\begin{array}{l}\text { «ce ton mari » } \\
\text { «ces tes } \\
\text { enfants » }\end{array}$ \\
\hline
\end{tabular}

Nous avons remarqué un nombre d'adverbes ajoutés par le traducteur, encore qu'ils soient assez peu diversifiés. La forme la plus fréquente est «normalnie » (normalement). Dans la plupart des cas, le mot est employé dans le sens qu'il prend uniquement dans le registre familier, à savoir «tout simplement », ce qui intensifie certainement l'effet de la langue orale.

\begin{tabular}{|l|l|l|l|}
\hline $\begin{array}{c}\mathbf{N}^{\mathbf{0}} \\
\text { de } \\
\text { page }\end{array}$ & \multicolumn{1}{|c|}{ Texte original } & $\begin{array}{l}\mathbf{N}^{\mathbf{0}} \\
\text { de } \\
\text { page }\end{array}$ & \multicolumn{1}{|c|}{ Texte de la traduction } \\
\hline 20 & $\begin{array}{l}\text { Quand le soleil a eu disparu, } \\
\text { les oiseaux, les criquettes, } \\
\text { pis les grenouilles ont } \\
\text { recommencé leur vacarme, } \\
\text { tout d'un coup, comme si } \\
\text { quelqu'un avait rallumé le } \\
\text { radio! }\end{array}$ & 4 & $\begin{array}{l}\text { A jak słońce znikło już całkowicie, to } \\
\text { ptaki, } \\
\text { świerszcze i zaby znowu zaczęły } \\
\text { hałasować. Od razu. Normalnie jakby ktoś } \\
\text { włączył radio! }\end{array}$ \\
\hline 82 & $\begin{array}{l}\text { T'étais heureuse comme } \\
\text { t'étais, Madeleine, pis au } \\
\text { fond, j'devais être un peu } \\
\text { jalouse de toi... }\end{array}$ & $\begin{array}{l}\text { Byłaś szczęśliwa, cieszyłaś się z tego, co } \\
\text { miałaś, Madeleine. A ja chyba tak } \\
\text { najnormalniej w świecie ci zazdrościłam... }\end{array}$ \\
\hline
\end{tabular}




\begin{tabular}{|l|l|l|l|}
\hline 90 & $\begin{array}{l}\text { D'habitude j’ai peur du noir, } \\
\text { mais là, j’aimerais ça rentrer } \\
\text { dedans... }\end{array}$ & 41 & $\begin{array}{l}\text { Zazwyczaj boję się ciemności. Ale tutaj, to } \\
\text { normalnie chciałabym w nią wejść... }\end{array}$ \\
\hline
\end{tabular}

Quant aux expressions phraséologiques (au sens large du terme), la question paraît un peu plus délicate, puisque ce sont des unités difficilement traduisibles. Cela justifie quelques divergences entre l'original et la version polonaise : il est tout à fait évident que les expressions phraséologiques n'apparaissent pas toujours aux mêmes endroits.

\begin{tabular}{|c|c|c|c|c|}
\hline $\begin{array}{l}\mathrm{N}^{\mathrm{o}} \mathrm{de} \\
\text { page }\end{array}$ & Texte original & $\begin{array}{l}N^{o} \\
\text { de } \\
\text { page }\end{array}$ & $\begin{array}{l}\text { Texte de la } \\
\text { traduction }\end{array}$ & $\begin{array}{l}\text { Traduction } \\
\text { littérale du } \\
\text { polonais en } \\
\text { français }\end{array}$ \\
\hline 17 & $\begin{array}{l}\text { Au même moment, joviale, } \\
\text { chantonnante et maigre comme un } \\
\text { barreau de chaise, Albertine à } 50 \\
\text { ans... }\end{array}$ & 3 & $\begin{array}{l}\text { W tym samym } \\
\text { momencie, szcreśliwa, } \\
\text { podśsiewnjaca } i \\
\text { odchudzona } \\
\text { Albertyna-50 }\end{array}$ & $\begin{array}{l}\text { Au même moment, } \\
\text { heureuse, } \\
\text { chantonnante et } \\
\frac{\text { amaigrie, Albertine à }}{50 \text { ans... }}\end{array}$ \\
\hline 38 & $\begin{array}{l}\ldots \text { même si y ont peur de moi } \\
\text { comme du bonhomme sept } \\
\underline{\text { heures }, . . .}\end{array}$ & 14 & $\begin{array}{l}\text { Nawet jeśli się } \\
\text { mnie boją. }\end{array}$ & $\begin{array}{l}\text { Même s'ils ont } \\
\text { peur de moi. }\end{array}$ \\
\hline 76 & $\begin{array}{l}\text { J'ai cassé le moule de mère- } \\
\text { poule! }\end{array}$ & 34 & $\begin{array}{l}\text { Wyszłam z } \\
\text { szufladki, w której } \\
\text { uwięziła mnie } \\
\underline{\text { matka! }}\end{array}$ & $\begin{array}{l}\text { Je suis sortie du } \\
\text { tiroir où ma mère } \\
\text { m’a enfermée! }\end{array}$ \\
\hline
\end{tabular}

\begin{tabular}{|c|c|c|c|c|}
\hline $\begin{array}{l}\mathrm{N}^{\circ} \mathrm{de} \\
\text { page }\end{array}$ & Texte original & $\begin{array}{l}N^{o} \text { de } \\
\text { page }\end{array}$ & $\begin{array}{l}\text { Texte de la } \\
\text { traduction }\end{array}$ & $\begin{array}{l}\text { Traduction } \\
\text { littérale du } \\
\text { polonais en } \\
\text { français }\end{array}$ \\
\hline 33 & $\begin{array}{l}\text { Moi aussi, des fois, au } \\
\text { commencement... je devais } \\
\text { pas sentir ben ben bon... }\end{array}$ & 11 & $\begin{array}{l}\text { Tak... Ja też, } \\
\text { czasami, na } \\
\text { początku... Nie } \\
\text { pachniałam } \\
\text { fiołkami... }\end{array}$ & $\begin{array}{l}\text { Oui, Moi aussi, au } \\
\text { début, je ne sentais } \\
\text { pas les violettes... }\end{array}$ \\
\hline 63 & $\begin{array}{l}\text { C'est facile pour toi de dire } \\
\text { ça, y t'arrive jamais rien! }\end{array}$ & 27 & $\begin{array}{l}\text { Latwo ci mówić. Ty } \\
\text { jesteś w czepku } \\
\text { urodzona! }\end{array}$ & $\begin{array}{l}\text { C'est facile pour toi } \\
\text { de dire ça, tu es née } \\
\text { en bonnet }\end{array}$ \\
\hline 80 & Ca risque pas d'arriver. & 36 & Nie ma takiej opcji. & $\begin{array}{l}\text { Il n'y a pas une telle } \\
\text { option! }\end{array}$ \\
\hline
\end{tabular}


Le style des femmes se caractérise par une disposition à employer la narration à la première personne

Il est difficile de parler d'une propension du traducteur-homme à éviter le « je », si le texte de départ est composé dans sa totalité à la première personne; les quelques exemples de l'omission de «je » que nous avons trouvés ne nous paraissent pas significatifs à cet égard.

\begin{tabular}{|l|l|l|l|l|}
\hline $\begin{array}{l}\mathbf{N}^{\text {o de }} \\
\text { page }\end{array}$ & \multicolumn{1}{|c|}{ Texte original } & $\begin{array}{l}\boldsymbol{N}^{\mathbf{o}} \text { de } \\
\text { page }\end{array}$ & $\begin{array}{l}\text { Texte de la } \\
\text { traduction }\end{array}$ & $\begin{array}{l}\text { Traduction littérale du } \\
\text { polonais en français }\end{array}$ \\
\hline 20 & $\begin{array}{l}\text { J'trouve que tu parles } \\
\text { drôlement... }\end{array}$ & 4 & Dziwnie mówisz... & Tu parles drôlement... \\
\hline 56 & $\begin{array}{l}\text { Bartine, j'comprends } \\
\text { pas c'que tu veux dire? }\end{array}$ & 23 & $\begin{array}{l}\text { Berciu, o co ci } \\
\text { chodzi? }\end{array}$ & $\begin{array}{l}\text { Bartine, qu'est-ce que } \\
\text { tu veux dire? }\end{array}$ \\
\hline 68 & $\begin{array}{l}\text { C'est pas Thérèse que } \\
\text { je frappais, je pense, } \\
\text { c'est ... c'est toute la } \\
\text { vie... }\end{array}$ & 30 & $\begin{array}{l}\text { To nie Thérèse } \\
\text { biłam... Nie... To } \\
\text { całe moje życie... }\end{array}$ & $\begin{array}{l}\text { Ce n'est pas Thérèse que } \\
\text { je frappais... Non... C'est } \\
\text {... c'est toute ma vie... }\end{array}$ \\
\hline
\end{tabular}

Ce qui nous semble plus intéressant, ce sont des emplois qui peuvent manifester un point de vue masculin ou une certaine difficulté à saisir des subtilités dans la perception du monde propre aux femmes :

\begin{tabular}{|c|c|c|c|c|}
\hline $\begin{array}{l}\mathrm{N}^{\circ} \mathrm{de} \\
\text { page }\end{array}$ & Texte original & $\begin{array}{l}\mathbf{N}^{o} \\
\text { de } \\
\text { page }\end{array}$ & $\begin{array}{l}\text { Texte de la } \\
\text { traduction }\end{array}$ & $\begin{array}{l}\text { Traduction littérale du } \\
\text { polonais en français }\end{array}$ \\
\hline 16 & $\begin{array}{l}\text { Elle est un peu ronde, } \\
\text { mais très belle. }\end{array}$ & 2 & \begin{tabular}{|l|} 
Jest nieco \\
zaokraglona, ale \\
wciąż bardzo ładna
\end{tabular} & $\begin{array}{l}\text { Elle est un peu ronde, } \\
\text { mais toujours (encore) } \\
\text { très belle. }\end{array}$ \\
\hline 58 & $\begin{array}{l}\text { Albertine à } 50 \text { ans quitte } \\
\text { sa place et vient s'installer } \\
\text { prés d'Albertine à } 70 \text { ans. } \\
\text { Elle lui prend doucement la } \\
\text { main. }\end{array}$ & 24 & $\begin{array}{l}\text { Albertyna-50 } \\
\text { pozostawia swoje } \\
\text { dotychczasowe miejsce i } \\
\text { idzie uto zyý sie koto } \\
\text { Albertyny-70. Bierze } \\
\text { delikatnie jej reke. }\end{array}$ & $\begin{array}{l}\text { Albertine à } 50 \text { ans quitte sa } \\
\text { place et vient s'installer prés } \\
\text { d'Albertine à } 70 \text { ans. Elle } \\
\text { prend doucement sa main. }\end{array}$ \\
\hline 77 & $\begin{array}{l}\text { Je me suis habillée en } \\
\text { neuf, du pas cher mais } \\
\text { du beau, pis chus partie } \\
\text { me chercher une job! } \\
\text { Comprenez-vous c'que } \\
\text { ça veut-dire? Une job! } \\
\text { La liberté! }\end{array}$ & 34 & \begin{tabular}{|lr} 
Kupiłam & sobie \\
nowe & ubrania. \\
Tanie, ale & ładne. I \\
poszłam r rzukać \\
pracy! Rozumiecie \\
co to znaczy? \\
Pracy! Wolności!
\end{tabular} & $\begin{array}{l}\text { Je me suis acheté des } \\
\text { vêtements neufs. Bon } \\
\text { marché, mais beaux. Et } \\
\text { je suis allée chercher du } \\
\text { travail! Comprenez-vous } \\
\text { ce que ça veut-dire? Du } \\
\text { travail! De la liberté! }\end{array}$ \\
\hline
\end{tabular}


Dans le premier exemple cité, la différence entre l'original et la traduction concerne l'adverbe «wciąż» (toujours, encore) qui renforce l'idée d'opposition entre "être ronde » et «être belle » exprimée déjà par la conjonction «mais ». Le deuxième fragment semble illustrer ce que nous avons déjà mentionné plus haut, à savoir la divergence entre le langage des hommes «orienté sur les objets » et celui des femmes « orienté sur les personnes ». Ainsi, la différence entre " prendre quelqu'un par la main» et " prendre la main de quelqu'un » est peut-être peu importante au niveau du contenu du message, mais elle devient beaucoup plus pertinente sur le plan des relations entre les participants de la communication ${ }^{11}$. Dans le dernier passage, c'est la traduction du mot « volonté» qui a attiré notre attention. D’où vient la désinence du génitif («-i») qui reflète en polonais l'article partitif dont elle est dépourvue? Est-ce une simple inadvertance ou bien un moyen permettant d'éviter un nom se terminant par la combinaison consonantique « $-\hat{s} \boldsymbol{c}^{\prime}$ », assez difficile à prononcer dans une exclamation en position finale absolue? Quelle que soit la motivation, le résultat est que si, dans la version originale, le seul fait d'« aller chercher une job» témoigne d'une certaine liberté de l'héroïne, dans la version traduite, Albertyna est toujours à la recherche de sa liberté. Voilà une nuance qui n'échappe certainement pas à l'attention des femmes!

\section{En guise de conclusion : masculinisation ou traducteur « cannibaliste »}

La perspective que nous avons adoptée nous mène tout naturellement vers la réflexion concernant l'attitude du traducteur envers le texte traduit : est-il un traducteur piétiste ou cannibaliste? Autrement dit, tente-t-il de rester fidèle à l'original de peur d'y changer quelque chose ou bien « dévore-t-il» l'original en vue d'offrir un texte nouveau? Il nous semble que les exemples que nous venons de présenter témoignent plutôt de l'attitude «cannibaliste» du traducteur. Nous nous garderions tout de même de l'accuser de trop s'ingérer dans le texte : il suit assez fidèlement la couche sémantique de l'original, plus précisément au niveau des significations référentielles. Mais d'autre part, comme nous l'avons vu dans les exemples cités ci-dessus, il ingère les significations pragmatiques du texte, c'est-à-dire le style propre aux femmes. Il nous semble qu'il «masculinise » légèrement le texte tremblayen, probablement de manière inconsciente, ce qui peut illustrer la thèse derridienne selon laquelle le fantasme sur la traduction sans traces s'avère irréel vu que le processus de traduction laisse toujours certaines traces qui effacent des différences entre l'original et la traduction. Comme le constate également Lori Chamberain, une traduction « muette » n'existe pas (399).

Si l'on revient au schéma de création-traduction évoqué par Lori Chamberlain (387) selon lequel l'écriture équivaut à l'originalité, à la masculinité et à la fidélité tandis que la traduction est associée, à l'inverse, au manque d'originalité, à la féminité et à l'infidélité, force est de constater que la version polonaise d'Albertine, en cinq temps ne se caractérise pas par la féminité, perçue non seulement en tant qu'élément soumis à l'original, mais aussi compris littéralement. Effectivement, dans la traduction de Jacek MulczykSkarżyński, on peut trouver de nombreux exemples témoignant de la différenciation du style selon le sexe. Ne serait-ce pas une illustration de la thèse énoncée par Bożena

11 Pour la notion de niveaux de communication cf. p.ex. Friedemann Schulz von Thun (2001). 
Tokarz selon qui " le traducteur écrit un texte d'autrui avec lui-même »" (12), c'est-àdire qu'il laisse toujours des traces de sa propre personnalité dans le texte traduit, notamment au niveau du style? Bien sûr, il ne faut pas oublier que «le sexe de la traduction» au niveau du langage est plus «une question de foi que de réalité » (Jermaszowa 120) et la recherche d'un élément féminin dans la traduction suscite toujours beaucoup de controverses. Et pourtant, dans le cas du texte tremblayen tout concourt à nous faire sentir que la parole féminine doit être absolument transmise en tant que telle vu que la version originale est susceptible d'éveiller des sentiments profonds de la part des femmes, ce dont témoignent les paroles de Martine Beaulne, une des metteures en scène de la pièce, celle d'ailleurs qui a été choisie par l'auteur luimême parce qu'il insistait que l'une des représentations soit montée par une femme : «Le cri d'Albertine, aucune femme ne peut être indifférente à ça » (citée par Paventi 74). Si l'on regarde le texte polonais dans l'optique de H. G. Gadamer, selon qui le traducteur devrait s'orienter vers le sens pour pouvoir transmettre tout ce qu'il devrait transmettre à travers la perspective de « son propre parler » (61), on peut constater avec un clin d'œil que Jacek Mulczyk-Skarżyński a bien apprivoisé cette règle en masculinisant l'original.

\footnotetext{
12 « (...) tłumacz cudzy tekst pisze sobą» (c'est nous qui traduisons).
} 


\section{Bibliographie}

Bednarczyk, Anna. "Płeć i przekład - uwagi o tematycznej nadinterpretacji i kontrowersyjnej praktyce"». Pté́ w przektadzie. Éd. Piotr Fast. KatowiceCzęstochowa : Śląsk 2006. 33-50.

Bukowski, Piotr, Heydel, Magda, réd.. Wspótczesne teorie prrekładu. Kraków : Znak 2009.

Camerlain, Lorraine : «Espaces sacrés : Albertine, en cinq temps et Messe solennelle pour une pleine lune d'été ». Jeu : revue de théâtre 79 (1996) : 125-129.

Cameron, Deborah. Feminism and Linguistic Theory. Londres : Macmillan, 1985.

Chamberlain, Lori. «Gender a metaforyka przekładu ». Wspótczesne teorie præękładu. .Éd. Piotr Bukowski, Magda Heydel. Kraków : Znak 2009. 386-402.

Chambers, Jack K. Sociolinguistic Theory: Linguistic Variation and its Social Signifiance. Oxford : Blackwell, 1995.

Cixous, Hélene. «Le rire de la Méduse ». L'Arc, 6 (1975) : 39-54.

Collet, Paulette. "Michel M. Tremblay: les leitmotive de son théâtre ». Le théatre canadien-français. Archives des Lettres canadiennes. Tome V. Éd. Paul Wyczynski.. Montréal : FIDES 1976. 597-793.

David, Gilbert, Lavoie, Pierre, réd. Le monde de Michel M. Tremblay. Tome I: théatre. Montréal/Carnières : Cahiers de Théâtre JEU/Editions Lansman, 2003.

Derrida, Jacques. De la grammatologie. Paris: Les Éditions de Minuit, 1967. . Eperons, Les Styles de Nietzsche. Paris: Flammarion, 1979.

Gadamer, Hans-Georg. "Człowiek i język ». Rozum, stowo, drieje. Szłkice wybrane. Éd. K. Michalski. Warszawa : PIW 2000. 52-62.

Godin, Jean Cléo : «Albertine, en cinq temps (1984). Albertine et la maison de l'enfance ». Le monde de Michel M. Tremblay. Tome I: théatre. Réd. David, Gilbert, Lavoie, Pierre. Montréal/Carnières : Cahiers de Théâtre JEU/Editions Lansman 2003.

Gumul, Ewa. «Kobieta-tłumacz, mężczyzna-tłumacz. Historia dwóch tekstów i kilku(dziesięciu) przekładów». Płé́ w præękładæie. Éd. Piotr Fast. KatowiceCzęstochowa : Śląsk 2006. 59-68.

Handke, Kwiryna. "Język a determinanty płci ». Jez̧yk a kultura, tom 9: Płé́ w jesylku $i$ kulturze. Réd. Janusz Anusiewicz, Kwiryna Handke. Wrocław : Wyd. Wiedza o kulturze 1994. 15-29.

Hay, Josianne. «Le casse-tête de la traduction du mot « gender» en français ». ILCEA, 3 (2002), : n. pag. Web. 8 juin 2010.

IMAS Report. «Zachowanie kobiet - mniej czy bardziej agresywne ?». Web. 29 mai 2011.

Jarniewicz, Jerzy. «Problematyka rodzaju w przekładzie literackim ». Warsqaty translatorskie. Workshop on translation. Lublin-Ottawa : TN KUL - SRG 2002. 7385.

Jermaszowa, Ira. «Jak rozpoznać « płeć» przekładu ? Na podstawie antologii Polskije poemy XX wieka Natalii Astafiewej i Władimira Britaniszskiego ». Plé́ w przęeładzie. Réd. Piotr Fast. Katowice-Częstochowa : Śląsk 2006. 119-128.

Kerbrat-Orecchioni, Catherine. "Converser au féminin ». La rhétorique au féminin. Éd. Annette Hayward. Québec : Éditions Nota Bene 2006. 33-81.

Labov William. Sociolinguistique. Paris : Minuit, 1976.

Labov William, "The Intersection of Sex and Social Class in the Course of Language Change», Language Variation and Change, vol. 2, 1990. 205-254.

Lévi-Strauss, Claude. "Le sexe des astres ». To honor Roman Jakobson. Essays on the occasion of his seventith birthday. 11 October 1966. Hague-Paris : Mouyon 1967. 1163-1170. 
Maliszewski, Julian. «Liryka Teresy Tomsi w przekładach Dorothei Müller-Ott, czyli rzecz o poszukiwaniu gender translation ». Pté́ w prrektadrie. Éd. Piotr Fast. Katowice-Częstochowa : Śląsk 2006. 151-176.

Osadnik, Wacław. "Asymetria językowa w opisie świata kobiet i mężczyzn na podstawie przysłów». Płé́ w przekłladrie. Réd. Piotr Fast. KatowiceCzęstochowa : Śląsk 2006. 91-102.

Paventi, Eza. «Albertine, en cinq voix ». Jeu 83 (juin 1997) : 70-74.

Przychodzeń, Janusz. «Les belles-soeurs en Pologne et au Québec : enjeux sociocritiques de la traduction et de la théâtralité ». L'Annuaire théatral : revue québécoise d'études théatrales, 27 (2000) : 120-133.

Ruprecht, Alvina. "Michel M. Tremblay et le temps perdu d'Albertine ». Canadian Drama / L'Art dramatique canadien. Vol. 12, 1 (1986) : 1-7.

Schulz von Thun, Friedemman. Sztuka rozmawiania. Trad. de l'allemand par Piotr Włodyga. Kraków : Wydaw. WAM, 2004.

Simon, Sherry. Gender in Translation. Cultural identity and the politics of transmission. London : Routledge, 1996.

Steiner, Georges. Après Babel. Une poétique du dire et de la traduction. Trad. de l'anglais par Lucienne Lotringer. Paris : Albin Michel, 1998.

Tokarz, Bożena. «Osoba w przekładzie». Biograficzne konteksty przekładu. Réd. Piotr Fast, Anna Kozak. Katowice : Śląsk 2002. 7-18.

Trudgill, Peter. "Social Identity and Linguistic Sex Differentiation ». On Dialect - Social and Geographic Perspectives. Éd. Peter Trudgill, Oxford : Blackwell 1983.

Von Flotow, Luise. «Feminist Translation : Contextes, Practices and Theories.» TTR : traduction, terminologie, rédaction, vol. 4, 2 (1991) : 69-84. Web. 24 avril 2011.

Yaguello, Marina. Les mots et les femmes. Essai d'approche socio-linguistique de la condition féminine. Paris : Payot, 1978. 